\title{
INVERSE HEAT CONDUCTION IN ANISOTROPIC AND FUNCTIONALLY GRADED MEDIA BY FINITE INTEGRATION METHOD
}

\author{
J. JIN ${ }^{1}$, J.L. ZHENG ${ }^{1}$, T. HUANG ${ }^{1}$, J.J. YANG ${ }^{1}$, H.S. WANG ${ }^{2}$, P.H.WEN ${ }^{2}$ \& J.M. LI $^{3}$ \\ ${ }^{1}$ School of Communication and Transportation Engineering, Changsha University of Science and \\ Technology, China. \\ ${ }^{2}$ School of Engineering and Materials Science, Queen Mary University of London, UK. \\ ${ }^{3}$ Department of Thermal Engineering, Tsinghua University, Beijing, China.
}

\begin{abstract}
Based on the recently developed finite integration method (FIM) for solving one and two dimensional ordinary and partial differential equations, this paper extends FIM to both stationary and transient heat conduction inverse problems for anisotropic and functionally graded materials with high degree of accuracy. Lagrange series approximation is applied to determine the first order of integral and differential matrices, which are used to form the system equation matrix for two and three dimensional problems. Singular Value Decomposition (SVD) is applied to solve the ill-conditioned system of algebraic equations obtained from the integral equation, boundary conditions and scattered temperature measurements. The convergence and accuracy of this method are investigated with two examples for anisotropic media and functionally graded materials.

Key words: finite integral method, integration matrix, inverse heat conduction, Lagrange series.
\end{abstract}

\section{INTRODUCTION}

Inverse heat conduction problems (IHCP) are used to estimate unknown quantities on the unreachable boundaries based on temperature and heat flux measurements in the analysis of physical problems in thermal engineering. As an example, the inverse heat conduction problem is used to compute the unknown temperature and heat flux at an unreachable boundary from scattered temperature measurements either at reachable interior points or boundary of the domain. In order to obtain the maximum information regarding the physical problem under study, IHCP enables a much closer collaboration between experimental and theoretical researchers. Since the solutions may become unstable as a result of errors inherent the measurements the difficulties encountered in the solution of IHTP should be recognized.

IHCP is mathematically classified as ill-posed in a general sense and is main reason that inverse problems were initially taken as not of physical interest. Traditional computational methods including the finite element method, boundary element method and meshless method for well-posed direct problems fail to produce acceptable solutions to these kinds of inverse problems. Several techniques have been proposed for solving a one-dimensional IHCP [1-5]. Applications with difference techniques can be found by the finite difference [6,7], boundary element $[8,9]$ and finite elements $[10,11]$ for two-dimensional IHCP. More recently, the method of fundamental solution $[12,13]$ and meshless methods have been developed to IHCP in two and three dimensions such as meshless local Petrov-Galerkin method [14, 15], which have recently attracted great attention in science and engineering communities for numerically solving time-dependent problems.

Despite the great success of the finite element method, boundary element method and meshless methods, there is still a need for developing numerical schemes for multi-dimensional IHCP. Recently, Wen and his colleagues [16-18] developed the Finite Integration Method (FIM) for solving one and two-dimensional, differential equation problems and 
demonstrated its applications to non-local elasticity problems. It has been shown that the FIM gives much higher degree of accuracy than the Finite Difference Method (FDM) and the Point Collocation Method (PCM). In this paper, the FIM is further extended to solve IHCP for anisotropic media and functionally graded materials.

\section{FORMULATION OF INVERSE HEAT CONDUCTION PROBLEMS}

We assume that in engineering, the thermal conductivities $\lambda_{i k}(\mathbf{x})$ are directionally dependent properties of an anisotropic material. All the properties of the material could be dependent on the spatial coordinates in a non-homogeneous material. The governing equation for steady heat conduction in anisotropic and continuously non-homogeneous media is given by the following partial differential equations with spatially variable properties:

$$
\begin{array}{lr}
q_{i, i}(\mathbf{x})=w(\mathbf{x}), & \mathbf{x}(x, y) \in \Omega \\
q_{i}(\mathbf{x})=-\lambda_{i k}(\mathbf{x}) T_{, k}(\mathbf{x}) & \\
\alpha \bar{T}(\mathbf{x})+\beta \bar{q}_{i}(\mathbf{x}) n_{i}=g(\mathbf{x}), & \mathbf{x}(x, y) \in \Gamma
\end{array}
$$

where $\mathrm{T}$ denotes the temperature distribution and $w(\mathbf{x})$ represents the heat source term, the first term is the rate of internal energy with time, the second term $q_{i}(\mathbf{x})$ is the heat flux vector, $\lambda_{i k}(\mathbf{x})$ is the thermal conductivity tensor, coefficients $\alpha$ and $\beta$ are constants, $n_{i}$ is the unit outward normal to the boundary. In addition, $T_{0}(\mathbf{x})$ is a given initial temperature in the domain and $g(\mathbf{x}, t)$ gives the boundary value. For inverse heat conduction problem, it is convenient to assume that the boundary $\Gamma$ consists of three parts $\Gamma=\left(\Gamma_{u} \cup \Gamma_{q} \cup \Gamma_{\sigma}\right)$ and the boundary conditions are given as

$$
\begin{array}{lll}
T(\mathbf{x})=\bar{T}(\mathbf{x}) & \mathbf{x} \in \Gamma_{u}, P_{T} \quad \text { (Dirichlet b.c. and Pint temperature) } \\
q(\mathbf{x})=q_{i}(\mathbf{x}) n_{i}(\mathbf{x})=\bar{q}(\mathbf{x}) & \mathbf{x} \in \Gamma_{q} \quad(\text { Neumann b.c. })
\end{array}
$$

where $\bar{T}(\mathbf{x})$ and $\bar{q}(\mathbf{x})$ are temperature and heat flux specified on the boundary. The IHCP to be investigated in this paper is to determine the temperature on the unreachable boundary $\Gamma_{\omega}$ from given Dirichlet data on $\Gamma_{u}$, Neumann data on $\Gamma_{q}$ and scattered temperature measurements $T_{P}(\mathbf{x})$ at some points $P_{T}$ either in the domain $\Omega$ or on the Neumann data boundary $\Gamma_{q}$.

\section{FIM USING LAGRANGE SERIES}

The simplest computation scheme for integration was introduced in Refs. [16, 17] and named as the Ordinary Linear Approach (OLA). For convenience of analysis in the following sections, coordinates $\mathrm{x}$ and $\mathrm{y}$ are used to replace $x_{1}$ and $x_{2}$, respectively, and subscripts $i$ or $k$ presents the nodal number. Consider an integral and apply trapezoidal integral rule

$$
U\left(x_{k}\right)=\int_{0}^{x_{k}} u(\xi) d \xi=\sum_{i=1}^{k} a_{k i} u\left(x_{i}\right)
$$

For trapezoidal rule, the coefficients are

$$
\begin{aligned}
& a_{1 i}=0, \\
& a_{k i}=\left\{\begin{array}{cc}
h, & i=1, \\
0.5 h, & i=2,3, \ldots, k-1, \\
0.5 h, & i=k, \\
0, & i>k,
\end{array}\right.
\end{aligned}
$$


where $x_{i}=(i-1) h, h=a /(N-1), i=1,2, \ldots, N$, are nodal points in [0,a], and $x_{1}=0, x_{N}=a$. Note that (3) can be written in a matrix form as

$$
\mathbf{U}=\mathbf{A} \mathbf{u}
$$

where $\mathbf{U}=\left[U_{1}, U_{2}, \ldots, U_{N}\right]^{T}, \mathbf{u}=\left[u_{1}, u_{2}, \ldots, u_{N}\right]^{T}$, and the first order of integration matrix

$$
\mathbf{A}=\left(a_{k i}\right)=\Delta\left(\begin{array}{cccccc}
0 & 0 & 0 & 0 & 0 & 0 \\
1 / 2 & 1 / 2 & 0 & 0 & 0 & 0 \\
1 / 2 & 1 & 1 / 2 & 0 & 0 & 0 \\
1 / 2 & 1 & 1 & 1 / 2 & 0 & 0 \\
\ldots & \ldots & \ldots & \ldots & \ldots & \ldots \\
1 / 2 & 1 & 1 & 1 & 1 & 1 / 2
\end{array}\right)_{N \times N}
$$

and $U_{i}=U\left(x_{i}\right), u_{i}=u\left(x_{i}\right)$ are the values of integration and the integral function respectively at each nodes.

The first order of integral matrix A in eqn (6) can be evaluated using the Lagrange interpolation series. The integral function is approximated as Ref. [17]

$$
u(x)=\sum_{k=1}^{N} c_{k} x^{k-1}
$$

where the coefficients $\left\{c_{k}\right\}_{k=1}^{N}$ are defined by

$$
\left[\begin{array}{ccccc}
1 & x_{1} & x_{1}^{2} & \ldots & x_{1}^{N-1} \\
1 & x_{2} & x_{2}^{2} & \ldots & x_{2}^{N-1} \\
\ldots & \ldots & \ldots & \ldots & \ldots \\
1 & x_{N} & x_{N}^{2} & \ldots & x_{N}^{N-1}
\end{array}\right]\left(\begin{array}{c}
c_{1} \\
c_{2} \\
\ldots \\
c_{N}
\end{array}\right)=\left(\begin{array}{c}
u_{1} \\
u_{2} \\
\ldots \\
u_{N}
\end{array}\right)
$$

or the integral function can be written as

Therefore, we have

$$
u(x)=\sum_{j=1}^{N} \prod_{\substack{k=1 \\ k \neq j}}^{N} \frac{\left(x-x_{k}\right)}{\left(x_{j}-x_{k}\right)} u_{j}
$$

$$
U\left(x_{i}\right)=\int_{0}^{(i-1) h} u(\xi) d \xi=\sum_{k=1}^{N} \frac{c_{k}}{k} x_{i}^{k}=\sum_{k=1}^{N} \frac{c_{k}}{k}(i-1)^{2} h^{2} i=1,2, \ldots, N
$$

For different boundary conditions, the first and second order of derivatives should be considered. Then for any points including two ends, from eqn (9), we have

$$
\frac{d u}{d x}=\sum_{k=2}^{N}(k-1) c_{k} x^{k-2} \text { and } \frac{d^{2} u}{d x^{2}}=\sum_{k=3}^{N}(k-1)(k-2) c_{k} x^{k-3} .
$$

From eqn (9), the first order derivative can also be written as

$$
\frac{d u}{d x}=u^{\prime}(x)=\sum_{j=1}^{N} u_{j} \prod_{k=1, k \neq j}^{N}\left(x_{j}-x_{k}\right)^{-1} \sum_{i=1}^{N} \prod_{k=1, k \neq j, k \neq i}^{N}\left(x-x_{k}\right)=\sum_{j=1}^{N} d_{j}(x) u_{j}
$$


Similar to the integration matrix, the first order derivative can be written, in matrix form, as

$$
\mathbf{D}=\mathbf{B u}
$$

where $\mathbf{D}=\left[u_{1}^{\prime}, u_{2}^{\prime}, \ldots u_{N}^{\prime}\right]^{T}, u_{k}^{\prime}=u^{\prime}\left(x_{k}\right)$ and matrix $\mathrm{B}$ is determined from eqn (12).

Thereafter, consider a multi-integral for one-dimensional problem

$$
U^{(2)}(x)=\int_{0}^{x} \int_{0}^{\xi_{2}} u\left(\xi_{1}\right) d \xi_{1} d \xi_{2}, \quad x \in[0, a]
$$

Applying the above OLA technique again for integral function $U^{(2)}(x)$, we have

$$
U^{(2)}\left(x_{k}\right)=\int_{0}^{x_{k}} \int_{0}^{\xi_{2}} u\left(\xi_{1}\right) d \xi_{1} d \xi_{2}=\sum_{i=0}^{k} \sum_{j=0}^{i} a_{k i} a_{i j} u\left(x_{i}\right)=\sum_{i=0}^{k} a_{k i}^{(2)} u\left(x_{i}\right) .
$$

In the same way for the first order integration Eq.(5), the multi-integral above can also be written in a matrix form as

$$
\mathbf{U}^{(2)}=\mathbf{A}^{(2)} \mathbf{u}=\mathbf{A}^{2} \mathbf{u}
$$

Therefore, we can extend this conclusion to any multi-layer integrals as

$$
\mathbf{U}^{(m)}=\mathbf{A}^{(m)} \mathbf{u}=\mathbf{A}^{m} \mathbf{u}
$$

In the same way, for higher order derivative to the function $\mathrm{u}$, we have

$$
\mathbf{D}^{(m)}=\mathbf{B}^{(m)} \mathbf{u}=\mathbf{B}^{m} \mathbf{u}
$$

\section{FIM FOR TWO DIMENSIONAL PROBLEMS}

For two-dimensional problems, consider a uniform distribution of collocation points as shown in Fig. 1, the integration matrix is defined as

$$
U_{x}(x, y)=\int_{0}^{x} u(\xi, y) d \xi, U_{x k}\left(x_{k}, y_{k}\right)=\int_{0}^{x_{k}} u\left(\xi, y_{k}\right) d \xi
$$

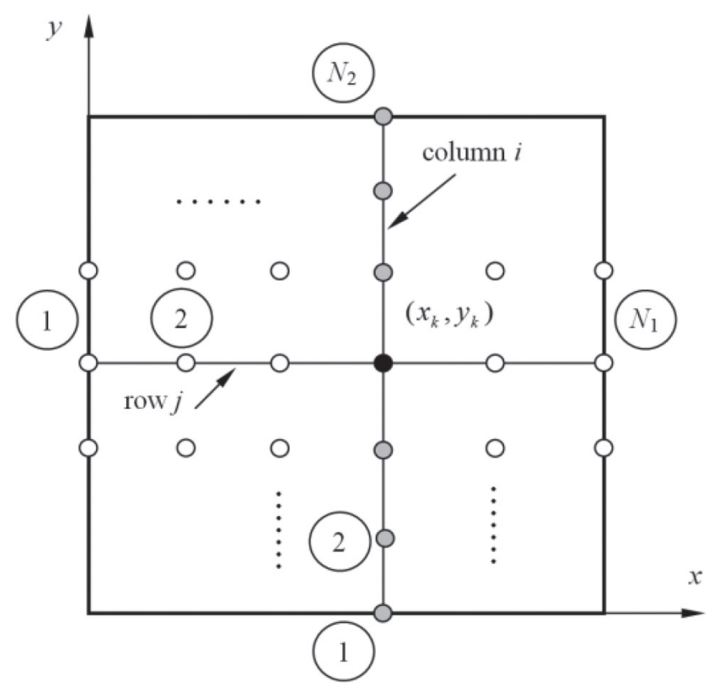

Figure 1:Uniform distribution of collocation points and the number of nodes. 
and the total number of points is $k=N_{1}(j-1)+i$, where $i$ and $j$ denote the number of column and the number of row, respectively. This numbering system is called the global number system. We can also express each nodal value of integration Eq. (19) in a matrix form as

$$
\mathbf{U}_{x}=\mathbf{A}_{x} \mathbf{u}
$$

where integral value $\mathbf{U}_{x}=\left[U_{x 1}, U_{x 2}, \ldots U_{x N}\right]^{T}$, nodal value function $\mathbf{u}=\left[u_{1}, u_{2}, \ldots, u_{M}\right]^{T}$ and $\mathrm{M}$ is the total number of collocation points $\left(M=N_{1} \times N_{2}\right)$ for grid shown in Fig. 1. For a rectangular domain, the first order integration matrix is

$$
\mathbf{A}_{x}=\underbrace{\left(\begin{array}{cccc}
\mathbf{A} & 0 & \ldots & 0 \\
0 & \mathbf{A} & 0 & 0 \\
\ldots & \ldots & \ldots & \ldots \\
0 & 0 & 0 & \mathbf{A}
\end{array}\right)}_{N_{2}}
$$

in which, $\mathbf{A}$ is the integration matrix for one-dimensional problem given in eqn (5) with dimension $N_{1} \times N_{1}$. Following the same procedure, the integration along y axis

$$
U_{y}(x, y)=\int_{0}^{y} u(x, \eta) d \eta, U_{y k}\left(x_{k}, y_{k}\right)=\int_{0}^{y_{k}} u\left(x_{k}, \eta\right) d \eta
$$

can be written in the matrix form as

$$
\mathbf{U}_{y}=\mathbf{A}^{\prime} \mathbf{u}
$$

in the local system for the collocation points, where $k=N_{2}(i-1)+j$. The first order integration matrix in the local system is

$$
\mathbf{A}^{\prime}=\underbrace{\left(\begin{array}{cccc}
\mathbf{A} & 0 & \ldots & 0 \\
0 & \mathbf{A} & 0 & 0 \\
\ldots & \ldots & \ldots & \ldots \\
0 & 0 & 0 & \mathbf{A}
\end{array}\right)}_{N_{1}}
$$

in which $\mathbf{A}$ is the integration matrix for one-dimensional problem given in eqn (5) with dimension $N_{2} \times N_{2}$. By a simple re-arrangement of the number of the nodes from the local number system to global number system, eqn (23) can be rewritten, in the global system, as

$$
\mathbf{U}_{y}=\mathbf{A}_{y} \mathbf{u} \text {. }
$$

For multi-integration in the two-dimensional problem in a rectangular domain, we consider the following integral with respect to coordinate $\mathrm{x}$

$$
U_{x}^{(2)}\left(x_{i}, y\right)=\int_{0}^{x_{i}} \int_{0}^{\xi_{2}} u\left(\xi_{1}, y\right) d \xi_{1} d \xi_{2}, \quad x_{i} \in[0, a], \quad y \in[0, b]
$$


where $a \times b$ is integral domain. Using the same procedure for one-dimension, we have

$$
U_{x}^{(2)}\left(x_{k}, y_{k}\right)=\int_{0}^{x_{k}} \int_{0}^{\xi_{2}} u\left(\xi_{1}, y_{k}\right) d \xi_{1} d \xi_{2}=\sum_{i=0}^{k} \sum_{j=0}^{i}\left(a_{k j}\right)_{x}\left(a_{j i}\right)_{x} u_{i}
$$

or in matrix form

$$
\mathbf{U}_{x}^{(2)}=\mathbf{A}_{x}^{2} \mathbf{u}
$$

where

$$
\mathbf{A}_{x}^{2}=\mathbf{A}_{x} \mathbf{A}_{x}=\underbrace{\left(\begin{array}{cccc}
\mathbf{A}^{2} & 0 & \ldots & 0 \\
0 & \mathbf{A}^{2} & 0 & 0 \\
\cdots & \ldots & \ldots & \ldots \\
0 & 0 & 0 & \mathbf{A}^{2}
\end{array}\right)}_{N_{2}}
$$

Similarly, we have multi-integration $U_{y}{ }^{(2)}(x, y)$ with respect to coordinate $\mathrm{y}$

and

$$
U_{y}{ }^{(2)}\left(x_{k}, y_{k}\right)=\int_{0}^{y_{k}} \int_{0}^{\eta_{2}} u\left(x_{k}, \eta_{1}\right) d \eta_{1} d \eta_{2}=\sum_{i=0}^{k} \sum_{j=0}^{i}\left(a_{k j}\right)_{y}\left(a_{j i}\right)_{y} u_{i}
$$

$$
\mathbf{U}_{y}{ }^{(2)}=\mathbf{A}_{y}^{2} \mathbf{u} \text {. }
$$

In addition, this method can be extended to multi-layers integration with two coordinates $\mathrm{x}$ and y as follow:

$U^{(m n)}\left(x_{k}, y_{k}\right)=\int_{0}^{x_{k}} \ldots \int_{0}^{\xi_{2}} \int_{0}^{y_{k}} \ldots \int_{0}^{\eta_{2}} u(\xi, \eta) \underbrace{d \xi_{1} \ldots d \xi_{m}}_{m \text {-layer }} \underbrace{d \eta_{1} \ldots d \eta_{n}}_{n \text {-layer }} \quad x_{k} \in[0, a], \quad y_{k} \in[0, b]$

and the nodal values of the above integration are obtained in the matrix form as

$$
\mathbf{U}^{(m n)}=\mathbf{A}_{x}^{m} \mathbf{A}_{y}^{n} \mathbf{u} .
$$

This method can be easily extended to multi-layer differentials with two coordinates $\mathrm{x}$ and $\mathrm{y}$ as

$$
\mathbf{D}^{(m n)}=\mathbf{B}_{x}^{m} \mathbf{B}_{y}^{n} \mathbf{u}
$$

\section{APPLICATION OF FIM TO TWO DIMENSION}

Example 1. Steady state heat conduction in an anisotropic square plate

Consider a square plate of width $a=b=1$, as shown in Fig. 2(a), with thermal conductivity tensor: $\lambda_{11}=\lambda_{22}=\lambda_{0}$ and $\lambda_{12}=\lambda_{21}=0.2 \lambda_{0}$. No heat source is considered, i.e. $w=0$. The analytical solution [15] is given as

$$
T^{*}(x, y)=x^{2}+x-5 x y
$$

which satisfies the stationary counterpart of governing eqn (1) for the considered thermal conductivity tensor. Then the boundary conditions are given as

$$
\bar{T}(x, 0)=x^{2}+x, \quad \bar{T}(x, 1)=x^{2}+x-5 x, \bar{q}(1, y)=4-5 y
$$




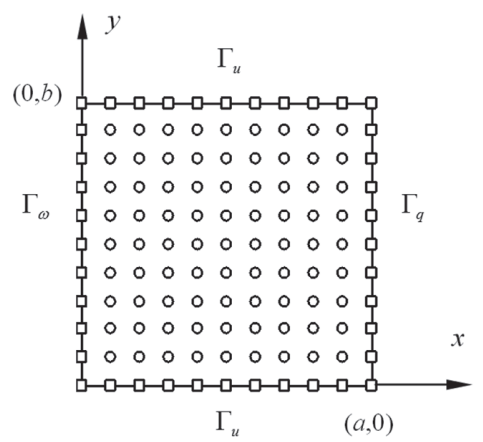

(a)

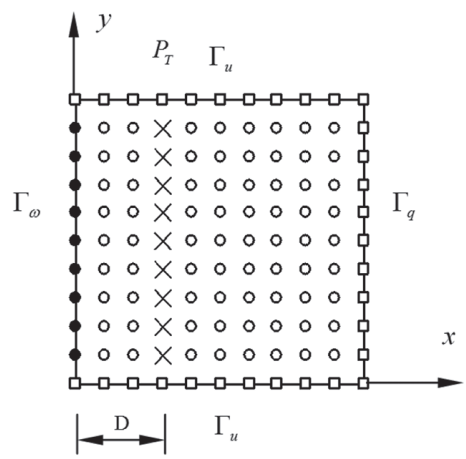

(b)

Figure 2: Distribution of collocation points: (a) internal and boundary points; (b) boundary conditions for inverse heat conduction problems, - unreachable boundary; $\square$ Neumann and Dirichlet boundary; $\times$ measurement points $P_{T}$ in domain, where $D / a$ indicates the gap.

and the scattered temperature measurement points are collected either on boundaries or in the domain, as shown in Fig. 2(b). The unreachable boundary is $x=0\left(\Gamma_{\omega}\right)$. Using integration matrix introduced in sections 3 or 4 , we have following governing equation in matrix form in terms of nodal values

$$
\left[\lambda_{11} \mathbf{A}_{y}^{2}+2 \lambda_{12} \mathbf{A}_{y} \mathbf{A}_{x}+\lambda_{22} \mathbf{A}_{x}^{2}\right] \mathbf{T}=\mathbf{X} \Psi_{y} \mathbf{f}_{1}+\Psi_{y} \mathbf{f}_{0}+\mathbf{Y} \Psi_{x} \mathbf{g}_{1}+\Psi_{x} \mathbf{g}_{0}
$$

w h e re $\mathbf{T}=\left\{T_{1}, T_{2}, \ldots, T_{M}\right\}^{T}, \mathbf{X}=\left\{x_{1}, x_{2}, \ldots, x_{M}\right\}, \mathbf{Y}=\left\{y_{1}, y_{2}, \ldots, y_{M}\right\}, \mathbf{f}_{k}=\left\{f_{1}^{k}, f_{2}^{k}, \ldots, f_{q}^{k}\right\}^{\mathrm{T}}$, $\mathbf{g}_{k}=\left\{g_{1}^{k}, g_{2}^{k}, \ldots, g_{p}^{k}\right\}^{\mathrm{T}},(k=0,1), \mathrm{q}$ and $\mathrm{p}$ are numbers of points to be used for interpolation of functions $f(y)$ and $g(x)$ collocated on $y$ and $x$ axes, respectively, $\Psi_{x}$ and $\Psi_{y}$ are shape function matrices of one-dimensional shape functions with respect to coordinates $x$ and $y$, respectively. Integral functions $f_{0}(y), f_{1}(y), g_{0}(x)$ and $g_{1}(x)$ can be interpolated in terms of the nodal values described in Ref. [17] with following two steps:

1. Determine the regions of functions $f(y)$ and $g(x)$, i.e. $\left[\bar{y}_{1}, \bar{y}_{q}\right],\left[\bar{x}_{1}, \bar{x}_{p}\right]$, and uniformly distributed points in these regions;

2. Determine one-dimensional shape function matrices $\Psi_{y}$ and $\Psi_{x}$

otherwise

$$
\begin{array}{ll}
f(y)=0 & \text { if } \bar{y}_{1}<y<\bar{y}_{n-1} \text { or } \bar{y}_{n}<y<\bar{y}_{q} \\
g(x)=0 & \text { if } \bar{x}_{1}<x<\bar{x}_{m-1} \text { or } \bar{x}_{m}<x<\bar{x}_{p}
\end{array}
$$

$$
\begin{aligned}
& f(y)=\frac{\bar{y}_{n}-y}{\bar{y}_{n}-\bar{y}_{n-1}} f_{n-1}+\frac{y-\bar{y}_{n-1}}{\bar{y}_{n}-\bar{y}_{n-1}} f_{n} \quad \text { if } \bar{y}_{n-1}<y<\bar{y}_{n} \\
& g(x)=\frac{\bar{x}_{m}-x}{\bar{x}_{m}-\bar{x}_{m-1}} g_{m-1}+\frac{x-\bar{x}_{m-1}}{\bar{x}_{m}-\bar{x}_{m-1}} g_{m} \quad \text { if } \bar{x}_{m-1}<x<\bar{x}_{m}
\end{aligned}
$$


For a regular distribution of nodes, we select $N_{1}=N_{2}=N, M=N^{2}, q=p=N$. Then the shape function matrices $\Psi_{x}=\Psi_{y}=\mathbf{I}$. It is obvious that the number of equations from boundary conditions is $L=4 \times N-4$. Thus the numbers of unknown nodal values $T_{i}$ is $\mathrm{M}$ and the numbers of nodal values of integral functions $f_{k}(y)$ and $g_{k}(x) 2 \times(p+q)=4 \times N$. SVD scheme is used to solve a set of linear algebraic equations [17]. The average error is defined as

$$
\varepsilon=\frac{1}{M_{\omega}} \sum_{i=1}^{M_{\omega}}\left|T_{i}-T_{i}^{*}\right|
$$

where $M_{\omega}$ is the number of nodes on the unreachable boundary, as shown in Fig. 2(b). The interior and boundary points are selected, as shown in Fig. 2(a) with $N_{1}=N_{2}=M_{\omega}=11$ and number of point measurements $M_{P}=N-2$. The average errors with different gaps between the unreachable boundary and measurement points for different noise levels are presented in Fig. 3. Apparently in the cases when $\sigma=0$, where $\sigma$ indicates the magnitude of noise, the numerical solutions are all close to the analytical solutions with maximum error of $10^{-3}(\%)$. For the cases of without noise, we take the measurements of temperature by $T\left(x_{P}, y_{P}\right)=$ $T^{*}\left(x_{P}, y_{P}\right)+\sigma \sin \left(\pi y_{P}\right)$. It is seen that the solutions are stable in the region of $D / a \leq 0.4$. When $D / a>0.5$, as expected, the error increases significantly since the measurement points are far away from the unreachable boundary. From Fig. 3, it can be observed that more accurate solutions could be obtained with smaller gaps. In addition, the convergence is observed with respect to the distribution density of nodes in the domain and on the boundary. When the interior and boundary points are selected as $N=17$, the differences between the numerical results for the two node distributions are seen to be very small.

Example 2. Two-dimensional steady state heat conduction problem

Consider steady state heat conduction in a quarter of disk (2D) with isotropic media, as shown in Fig. 4(a). The governing equation (1) in polar coordinate system is

$$
\frac{\partial^{2} T}{\partial r^{2}}+\frac{1}{r} \frac{\partial T}{\partial r}+\frac{1}{r^{2}} \frac{\partial^{2} T}{\partial \theta^{2}}=2 r^{2} \quad 1 \leq r \leq 2,0 \leq \theta \leq \pi / 2
$$

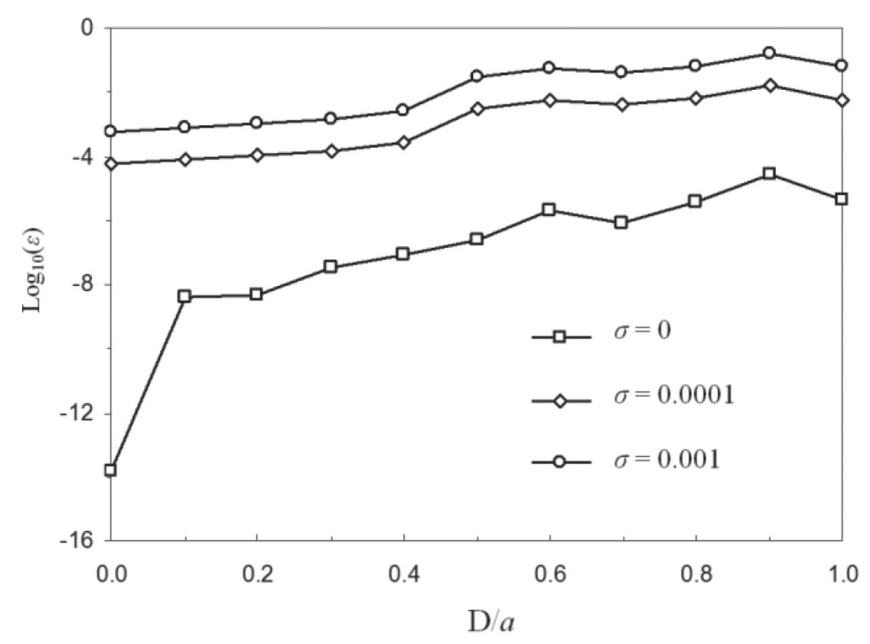

Figure 3: Average error versus the gap between unreachable boundary and measurement points with different levels of noise at measurement points. 


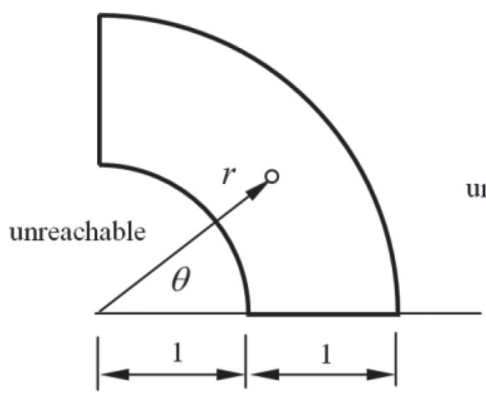

(a)

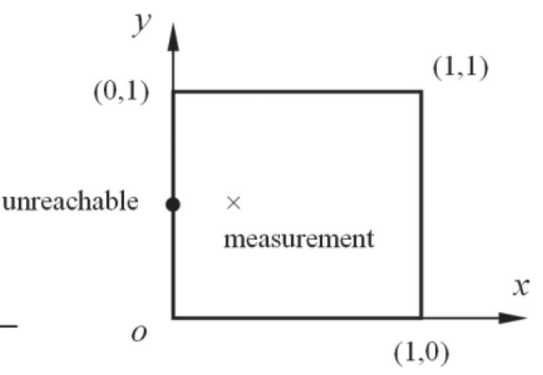

(b)

Figure 4: (a) Quarter of ring; (b) transformation of coordinates.

with the boundary conditions $T(r, 0)=T(r, \pi / 2)=0 ; T(1, \theta)=1 / 4 \sin ^{2} 2 \theta ; T(2, \theta)=4 \sin ^{2} 2 \theta$. The exact solution is obtained as $T^{*}(r, \theta)=r^{4} / 4 \sin ^{2} 2 \theta$. By coordinate transform, Eq. (41) can be normalised as

$$
\frac{\partial^{2} T}{\partial x^{2}}+\frac{\partial}{\partial x}\left(\frac{T}{1+x}\right)+\frac{T}{(1+x)^{2}}+\frac{4}{(1+x)^{2} \pi^{2}} \frac{\partial^{2} T}{\partial y^{2}}=2(1+x)^{2}
$$

where transformed coordinates $x=r-1$ and $y=\pi \theta / 2$ and the domain is transformed into a square as shown in Fig. 4(b). Using the integration matrix in section 4, we have following governing equation, in matrix form, in terms of nodal values

$$
\left[\mathbf{A}_{y}^{2}+\mathbf{A}_{y}^{2} \mathbf{A}_{x}+\mathbf{A}_{x}^{2} \mathbf{A}_{y}^{2} \mathbf{R}_{1}+\frac{4}{\pi^{2}} \mathbf{A}_{x}^{2} \mathbf{R}_{2}\right] \mathbf{T}=\mathbf{A}_{y}^{2} \mathbf{A}_{x}^{2} \mathbf{w}+\mathbf{X} \Psi_{y} \mathbf{f}_{1}+\Psi_{y} \mathbf{f}_{0}+\mathbf{Y} \Psi_{x} \mathbf{g}_{1}+\Psi_{x} \mathbf{g}_{0}
$$

where

$$
\mathbf{R}_{1}=\operatorname{diag}\left\{1 /\left(1+x_{i}\right\}, \mathbf{R}_{2}=\mathbf{R}_{1}^{2}, \mathbf{w}=\operatorname{diag}\left\{2\left(1+x_{i}\right)^{2}\right\}\right.
$$

in which the integral functions on the right hand side are defined in eqn (37). Same nodes distribution in Example 1 are used. The average relative error is defined as

$$
\varepsilon_{\mathrm{re}}=\frac{1}{M} \sum_{i=1}^{M}\left|T_{i}-T_{i}^{*}\right| / T_{\max }^{*}, \quad T_{\max }^{*}=T^{*}(2, \pi / 4)=4
$$

Variation of the logarithms of the average relative error $\varepsilon_{\text {re }}$ with different numbers of grid $N$ is shown in Fig. 5. For comparison the numerical solutions obtained by the finite difference method (FDM) is also given in Fig. 5. It is seen that the accuracy of FDM solutions increases linearly with increasing the number of nodes. As expected, the accuracy of the numerical solutions obtained by by FIM is much higher than that by FDM even for a small number of grid $N$. Secondly, we consider that the unreachable boundary $\Gamma_{\omega}$ is collocated at inner surface $(x=0)$. By changing $M$ to $M_{\omega}$ in eqn (45) and selecting the number of grid $N=11$, Fig. 6 shows the average errors over the unreachable boundary for different gaps D and different levels of noise at the points of measurement. To observe the effect of measurement noise, the 


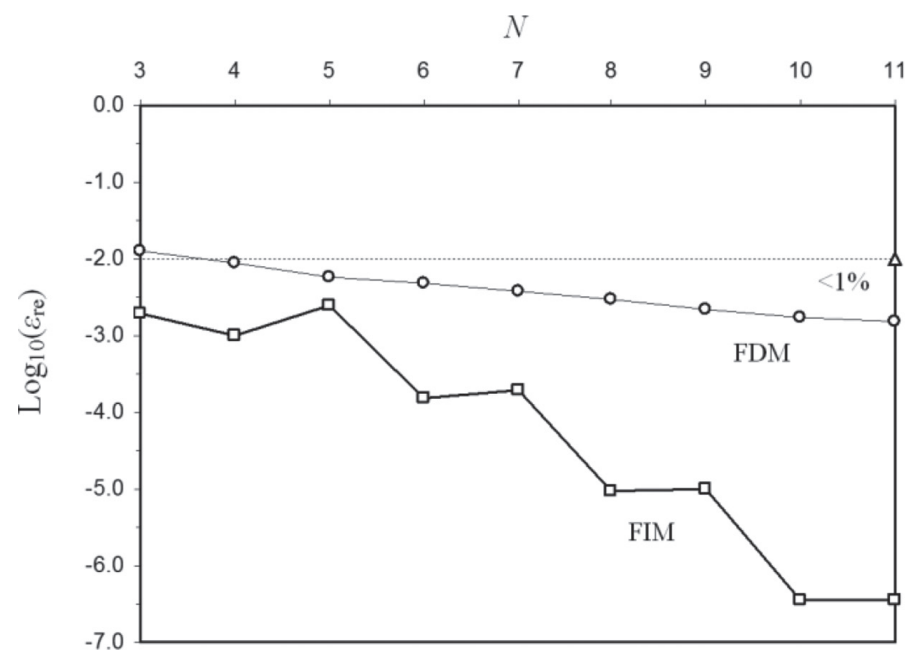

Figure 5: The logarithms of the average relative error versus the segment number $N$.

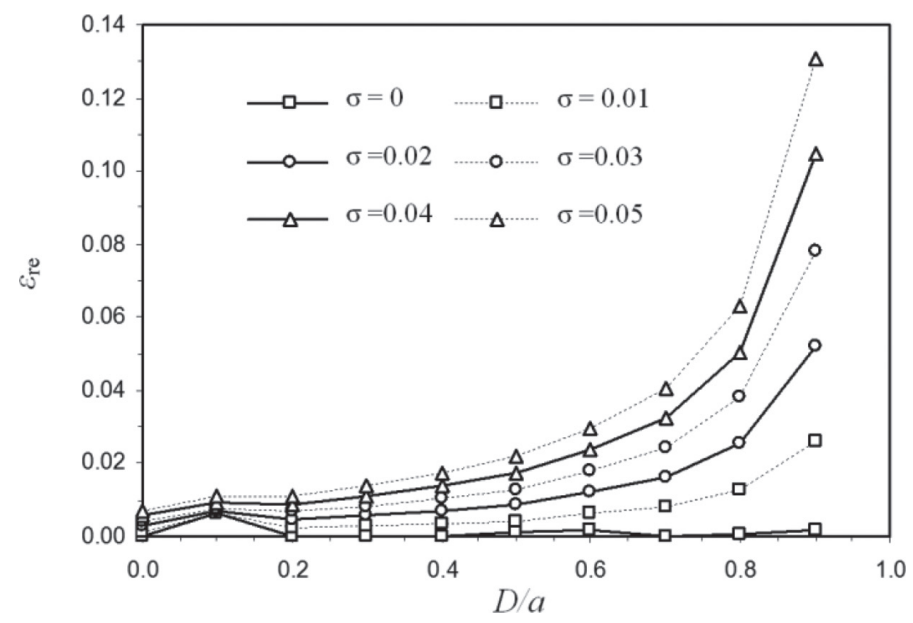

Figure 6: The average errors in the domain versus the gap between unreachable boundary and measurement points for different levels of noise.

measurements of temperature are taken by $T\left(x_{P}, y_{P}\right)=T^{*}\left(x_{P}, y_{P}\right)+\sigma \sin \left(\pi y_{P}\right)$. Apparently in the region of $D / a \leq 0.6$, it is seen that the temperature obtained on unreachable boundary is stable. However, when $D / a>0.7$, the error increases significantly with noise.

\section{CONCLUSIONS}

The finite integration method with Lagrange series expansion has been demonstrated for two and three dimensional inverse heat conduction problems for the first time. Anisotropic media and functionally graded materials are considered. Approximation by Lagrange series is used to determine the first order integration and derivative matrices. The Singular Value 
Decomposition (SVD) is applied to solve the ill-posed linear system of algebraic equations, derived from the governing equation, boundary conditions and scattered temperature measurements in the present work. The stability and accuracy were observed for inverse heat conduction problems in three examples. It has been shown that the finite integration method provides an efficient algorithm for solving inverse heat conduction problems. However, if the Dirichlet condition (measurements) and Neumann condition are specified at the same points of the boundary, the prediction of the temperature on the unreachable boundary becomes unstable with certain noises from measurement.

\section{REFERENCES}

[1] Jonas, P. \& Louis, A.K., Approximate inverse for a one-dimensional inverse heat conduction problem. Inverse Problems, 16, pp. 175-185, 2000. https://doi.org/10.1088/0266-5611/16/1/314

[2] Lesnic, D. \& Elliott, L., The decomposition approach to inverse heat conduction. Journal of Mathematical Analysis and Applications, 232, pp. 82-98, 1999. https://doi.org/10.1006/jmaa.1998.6243

[3] Liu, J., A stability analysis on Beck's procedure for inverse heat conduction problem. Journal of Computational Physics, 123, pp. 65-73, 1996. https://doi.org/10.1006/jcph.1996.0005

[4] Shen, S.Y., A numerical study of inverse heat conduction problems. Computers \& Mathematics with Applications, 38, pp. 173-188, 1999. https://doi.org/10.1016/s0898-1221(99)00248-5

[5] Hon, Y.C. \& Wei, T., A fundamental solution method for inverse heat conduction problem. Engineering Analysis with Boundary Elements, 28, pp. 489-495, 2004. https://doi.org/10.1016/s0955-7997(03)00102-4

[6] Guo, L. \& Murio, D.A., A modified space-marching finite-difference algorithm for the two-dimensional inverse heat conduction problem with slab symmetry. Inverse Problems, 7, pp. 247-259, 1991. https://doi.org/10.1088/0266-5611/7/2/008

[7] Khalidy, N.A., A general space marching algorithm for the solution of two-dimensional boundary inverse heat conduction problems. Numerical Heat Transfer Part B, 34, pp. 339-360, 1998. https://doi.org/10.1080/10407799808915062

[8] Chantasiriwan, S., An algorithm for solving multidimensional inverse heat conduction problem. International Journal of Heat and Mass Transfer, 44, pp. 3823-3832, 2001. https://doi.org/10.1016/s0017-9310(01)00037-0

[9] Kurpisz, K. \& Nowak, A.J., BEM approach to inverse heat conduction problems. Engineering Analysis with Boundary Elements, 10, pp. 291-729, 1992. https://doi.org/10.1016/0955-7997(92)90142-t

[10] Hsu, T.R., Sun, N.S., Chen, G.G. \& Gong, Z.L., Finite element formulation for twodimensional inverse heat conduction analysis. ASME Journal of Heat Transfer, 114, pp. 553-557, 1992. https://doi.org/10.1115/1.2911317

[11] Reinhardt, H.J., A numerical method for the solution of two-dimensional inverse heat conduction problems. International Journal for Numerical Methods in Engineering, 32, pp. 363-283, 1991.

https://doi.org/10.1002/nme.1620320209 
[12] Hon, Y.C. \& Wei, T., The method of fundamental solutions for solving multidimensional inverse heat conduction problems. Computer Modeling in Engineering \& Sciences, 7, pp. 19-32, 2005.

[13] Yu, G.X., Sun, J., Wang, H.S., Wen, P.H. \& Rose, J.W., Meshless inverse method to determine temperature and heat flux at boundaries for 2D steady-state heat conduction problems. Experimental Thermal and Fluid Science, 52, pp. 156-163, 2004.

https://doi.org/10.1016/j.expthermflusci.2013.09.006

[14] Atluri, S.N., The Meshless Method (MLPG) for Domain and BIE Discretizations, Tech Science Press Forsyth GA, 2004.

[15] Sladek, J., Sladek, V. \& Hon, Y.C., Inverse heat conduction problems by meshless local Petrov-Galerkin method. Engineering Analysis with Boundary Elements, 30, pp. 650-661, 2006.

https://doi.org/10.1016/j.enganabound.2006.03.003

[16] Li, M., Hon, Y.C., Korakianitis, T. \& Wen, P.H., Finite integration method for nonlocal elastic bar under static and dynamic loads. Engineering Analysis with Boundary Elements, 37(5), pp. 842-849, 2013.

https://doi.org/10.1016/j.enganabound.2013.01.018

[17] Wen, P.H., Hon, Y.C., Li, M. \& Korakianitis, T., Finite integration method for partial differential equations. Applied Mathematical Modelling, 37, pp. 10092-10106, 2013. https://doi.org/10.1016/j.apm.2013.05.054

[18] Li, M., Tian, Z.L., Hon, Y.C., Chen, C.S. \& Wen, P.H., Improved finite integration method for partial differential equations. Engineering Analysis with Boundary Elements, 64, pp. 230-236, 2016.

https://doi.org/10.1016/j.enganabound.2015.12.012 